\title{
Non linear half-order modeling of synchronous machine
}

\author{
S. Racewicz*, D. Riu**, N. Retière**, P. Chzran* \\ *Department of Power Electronics and Electrical Machines, Gdansk University of Technology, ul. Sobieskiego 7, \\ 80-216 Gdansk, Poland \\ **Laboratoire de Génie Electrique de Grenoble, G2Elab/Ense3, Domaine universitaire, BP 46, \\ 38402 Saint-Martin-d'Hères Cedex France
}

\begin{abstract}
Non integer order systems are used to model diffusion in conductive parts of electrical machines as they lead to more compact and knowledge models but also to improve their precision. Nevertheless, non integer order models have been established under linear conditions. It is than interesting to study the influence of saturation on such model parameters. In this article, a linear half-order impedance model of a ferromagnetic sheet deduced from the diffusion of the magnetic field is introduced. Then, a non linear half-order impedance model of the sheet is proposed. It is finally validated by performing $3 \mathrm{D}$ finite element simulations and experimentally verified on a $3 \mathrm{kVA}$ saturated synchronous machine.
\end{abstract}

\section{INTRODUCTION}

Current embedded generation requires to come back over the modelling of supply groups of various principles and power ranges in order to study the impact of their integration on the dynamic behaviour of systems as aeronautic systems. The current aim is then to improve modelling in order to optimize the control of these systems which are highly subjected to manifold disruptions or state uncertainties (load steps, saturations, time-delays and so on).

In this article, we have focused on synchronous machine modeling. It is then necessary not only to supply accurate models of machines over a wide range of frequencies, but also to include knowledge in models. Moreover, model order has to be reduced as robust control techniques lead also to high-order controllers [1]. The system order is then so high that classical dynamic studies like stability become difficult.

In most generators, as frequency increases, induced currents can no longer be neglected. Equivalent circuits of synchronous machines are then improved by including ladder elements with constant parameters [2,3]. However, as this effect is a distributed phenomenon described by partial differential equations, classical improved equivalent circuits must include in theory an infinite number of lumped and constant parameters.

In previous works, the authors have proposed an alternative way of modelling based on non integer order systems. These systems have then been used to model diffusion in conductive parts of electrical machines as they lead to more compact and knowledge models but also to improve their precision [4]. Nevertheless, non integer order models rest valid for linear cases. It is than interesting to study the influence of saturation on such model parameters as saturation strongly influences the field current and the load angle for each load behaviour condition [5].

As robust control can be applied to non linear processes, the non-linearity may be taken into account as an uncertainty which influences the control design. The proposed model would then present three retrieved benefits: a knowledge model with a much reduced order and a global simplicity compared to finite element models, magnetic equivalent circuit, artificial neural networks or even models based on cross-magnetization which lead to $\mathrm{d}$ and $\mathrm{q}$ axes inductances which depend on the $\mathrm{q}$ and $d$ armature currents $[5,6,7]$.

In this paper, the authors have focused on the modelling of saturation of ferromagnetic sheet using half-order systems. Following an overview of non integer order systems, a linear half-order impedance model of a ferromagnetic sheet deduced from the diffusion of the magnetic field is briefly introduced as this work has been already detailed in previous works [4]. Then, from physical considerations, a non linear half-order impedance model of the sheet is proposed taking into account of saturation and diffusion both. The proposed model is finally validated by performing $3 \mathrm{D}$ finite elements simulations and with tests performed on a synchronous machine of $3 \mathrm{kVA}$ working in saturated conditions.

\section{PRESENTATION OF NON-ORDER SYSTEMS}

Some linear systems can be represented by constant parameter differential equations which order $n$ is non integer or even complex. Such systems are used for complex systems control, non-Newtonian fluids modeling, fractal structures or distributed parameters systems which are represented by partial differential equations [8].

A normalized system of order 1 can be characterized by the following transmittance:

$$
T_{1}(\omega)=\frac{1}{1+j \frac{\omega}{\omega_{0}}}=\frac{1}{1+u}
$$

where $\omega_{0}$ is the cut-off frequency of the system and $u$ the normalized frequency (in per unit). 


\section{IEMDC2009-10981}

A non integer order system of order $\mathrm{n}$ is very similarly characterized by equation (2). As an instance, Fig. 1 describes the Bode diagram of both half-order and integer order systems presented above.

$$
T_{n}(\omega)=\frac{1}{\left(1+j \frac{\omega}{\omega_{0}}\right)^{n}}=\frac{1}{(1+u)^{n}}
$$
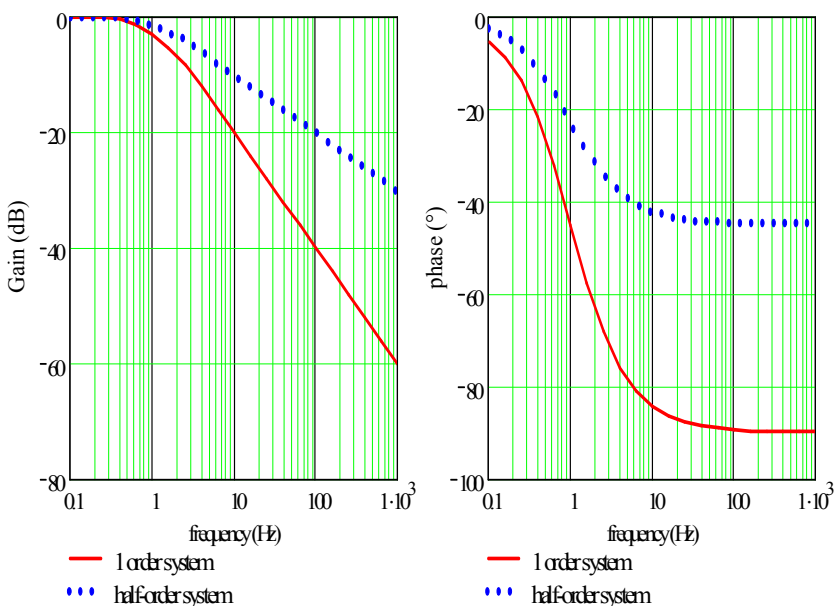

Fig. 1: Bode diagrams of first and half-order systems

Therefore, it should be noticed that the gain asymptote of a half-order system decreases by $10 \mathrm{~dB}$ along a decade and the phase asymptote is $-45^{\circ}$.

The main property of non integer systems is their compactness, as they include at the same time slow and fast dynamics; their time response can then be divided in two phases; first, the system starts to answer very quickly to solicitations, then it spends a long time to reach the end value.

In electrical engineering, non integer order systems have been used to model classical electrical machines, as induction machines [9], synchronous machines [4] or transformers. All frequency models presented as equivalent circuits are globally of reduced order compared to classical integer order ones thanks to property presented above. They also found an application in electrochemical devices modeling such as batteries [10], supercapacitors or fuel cells [11] taking into account of the species diffusion in electrochemical double layers.

In another way, non-integer order systems also allow to link geometrical properties of large electrical networks with their frequency characterization. This allows to obtain extremely compact equivalent dynamics of complex systems that can be very interesting for the analysis and network planning.

\section{HALF-ORDER MODELLING OF SYNCHRONOUS MACHINES IN LINEAR CONDITIONS}

First, the non integer order modeling is introduced with the modeling of a simple rectangular ferromagnetic sheet. Then, non integer models of a synchronous machine are presented from physical considerations.

\section{A. Ferromagnetic sheet and rotor bar modeling}

The radius of a machine's rotor is supposed to be sufficiently large for being represented as a sheet (Fig. 2).

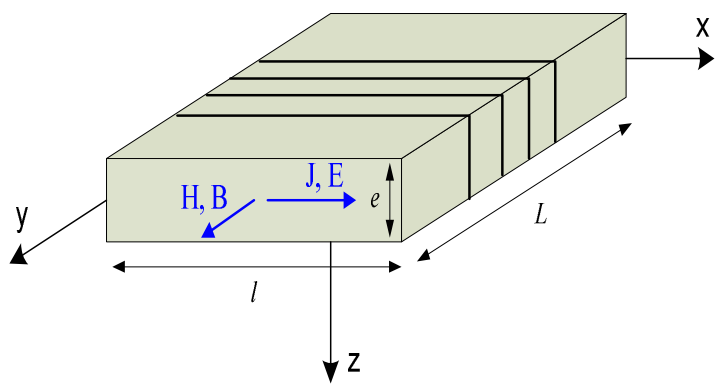

Fig. 2: Studied ferromagnetic sheet

This sheet is characterized by a permeability $(\mu)$, a resistivity $(\rho)$ and surrounded by a n-turns field winding. Classical analytical model of its impedance can be expressed by (3) [12]:

$$
Z_{\text {ana }}=j \omega L_{0} \frac{\tanh (\bar{\alpha})}{\bar{\alpha}}
$$

with:

$$
L_{0}=\frac{\mu . n^{2} . e . l}{L} \text { and } \bar{\alpha}^{2}=j \omega \frac{\mu}{\rho}\left(\frac{e}{2}\right)^{2}
$$

$L_{0}$ is the DC inductance of the sheet.

From this analytical model, an integer order approximation of this impedance from serial decomposition of tangent hyperbolic function can then be classically used in simulation software [2,12].

In parallel, from a second-order limited development of tangent hyperbolic function given by:

$$
\tanh (x)=\frac{\sinh (x)}{\cosh (x)} \underset{x \rightarrow 0}{\approx} \frac{x}{1+\frac{x^{2}}{2}} \underset{x \rightarrow 0}{\approx} \frac{x}{\sqrt{1+x^{2}}}
$$

a non integer approximation of the analytical impedance is given by [4]:

$$
Z_{\text {inductive }}^{1 / 2}(\omega)=\frac{j L_{0} \omega}{\sqrt{1+j \frac{\omega}{\omega_{0}}}}
$$

with: 


$$
\omega_{0}=\frac{\rho}{\mu}\left(\frac{2}{e}\right)^{2}
$$

$\omega_{0}$ is the DC inductance of the sheet.

Equation (6) represents an inductive half-order impedance thanks to its DC behaviour. Fig. 3 allows to compare analytical and half-order models on a large frequency range. It can be noticed that this half-order models is very relevant compared with the analytical expression (3).
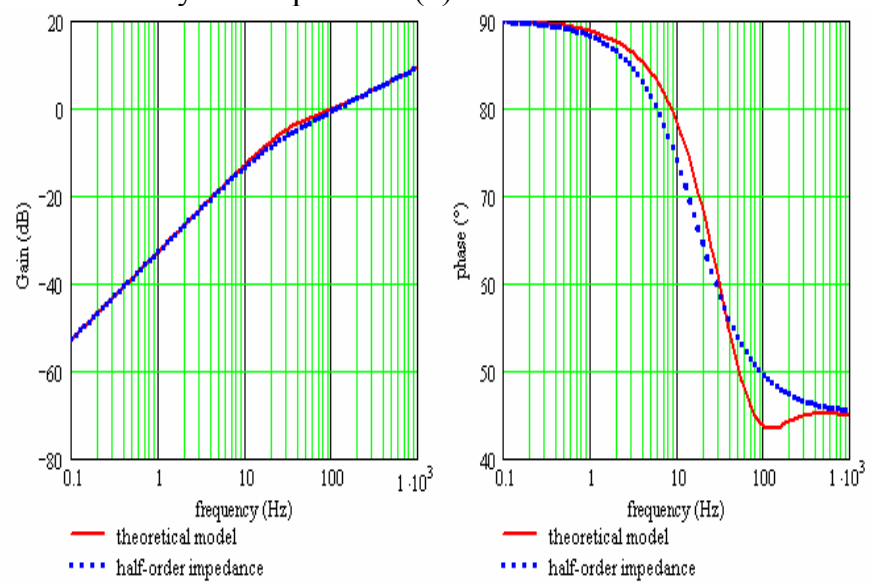

Fig. 3: Analytical and half-order expressions of sheet's impedances

The same approach is used for damper bars modelling. This leads to a resistive half-order impedance which parameters R0 and $\omega_{0}$, the DC resistance and cut-off frequency of a rectangular conductive bar, are directly to constructive parameters of the machine.

$$
Z_{\text {resistive }}^{1 / 2}(\omega)=R_{0} \sqrt{1+j \frac{\omega}{\omega_{0}{ }^{\prime}}}
$$

\section{B. Non-integer equivalent circuit of synchronous machine under linear conditions}

Finally, half-order linear impedances can be included in the Park equivalent circuits of synchronous generators (Fig. 4) following some physical conditions $[3,4]$. Then, for $d$-axis modelling, no induced currents expand in armature windings or field windings for low frequencies; they are then modelled by constant parameters: $\left(r_{d}, l_{d}\right)$ for stator and $\left(r_{f}, l_{f}\right)$ for field windings. Then, a constant inductance (lad) is introduced to model the stored energy in the air gap. Finally, eddy-currents development in massive parts of the rotor and in damper bars are modelled by an inductive $\left(Z_{\text {inductive }}^{1 / 2}\right)$ and a resistive $\left(Z_{\text {resistive }}^{1 / 2}\right)$ impedances respectively described in (6) and (8).

The same considerations are taken for the q-axis except that there are no excitation windings. The corresponding equivalent circuits are described on Fig. 4.
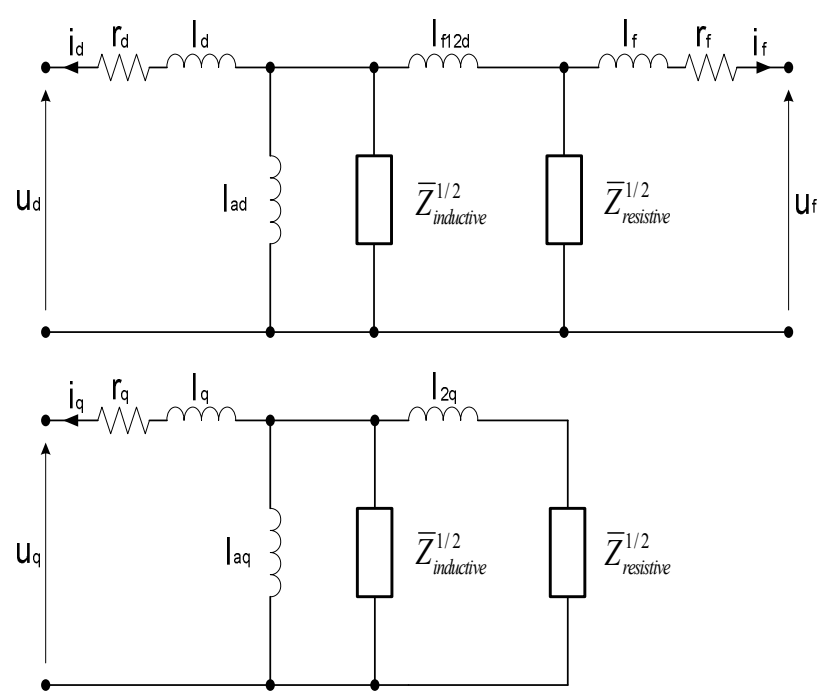

Fig. 4: Non integer order $\mathrm{d}$ and q-axis equivalent circuits of an alternator

The circuit parameters are then evaluated by curve-fitting from operational impedances issued from StandStill Frequency Response tests (SSFR) [13]. The results of identification are in good agreement with the expected values: angular frequencies of several Hertz for the damper windings or some miliHertz for the rotor [4]. Moreover, model order has significantly decreased with, for instance, an equivalent circuit in q-axis which includes only 8 parameters whereas any integer order includes more then 13 for an equivalent precision.

\section{NON-LINEAR MODELLING OF FERROMAGNETIC SHEET}

The authors propose a new model of the inductive half-order impedance taking into account non-linear conditions. Indeed, in electrical machines, magnetic saturation of ferromagnetic pieces influences mainly the value of the inductances [14].

First, it is important to note that we are only interested in small-signal modelling for linear control operations. Then, we look at small dynamic variations of electrical variables (current or voltage) for a given magnetic state of the machine.

The frequency structure of the model presented in equation (6) is kept quite identical, but parameters $L_{0}$ and $\omega_{0}$ vary with the current $i$ as shown below:

$$
Z_{\text {inductive,sat }}^{1 / 2}(\omega, i)=\frac{j \cdot L_{0}(i) \cdot \omega}{\sqrt{1+j \frac{\omega}{\omega_{0}(i)}}}
$$

The evolution of $\mathrm{L}_{0}$ and $\omega_{0}$ with the current should be physically significant with a decrease of $\mathrm{L}_{0}$ and an increase of $\omega_{0}$ with current growth as apparent permeability decreases with saturation. 
In order to validate the proposed model, finite elements simulations of the ferromagnetic sheet have been done with Flux $($ ) software. The impedances were calculated over the range of frequency from $0.01 \mathrm{~Hz}$ to $1000 \mathrm{~Hz}$. An amplitude of a current in a one turn winding corresponded to the values $0.01,0.1,1,10$ and $20 \mathrm{~A}$, which gave a magnetic field of 0.05 , $0.5,5,49.7$ and $99.4 \mathrm{~A} / \mathrm{m}$ respectively.
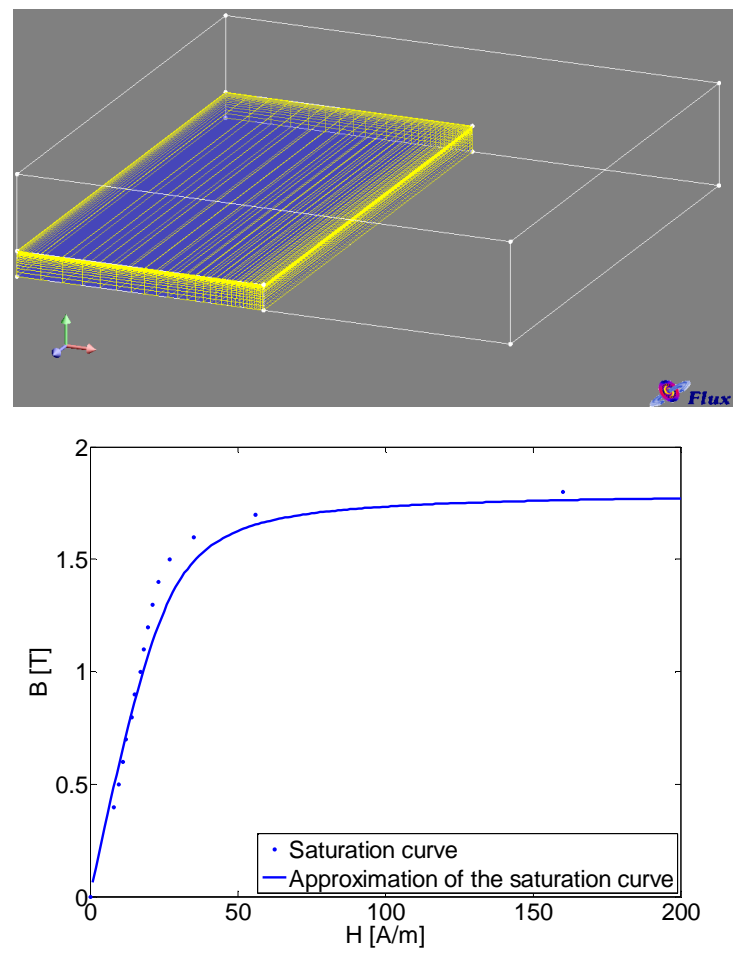

Fig.5. Modelled ferromagnetic sheet with its saturation curve

The gain of ferromagnetic sheet impedance is then plotted on Fig. 6.

In a second step, parameters $L_{0}(i)$ and $\omega_{0}(i)$ can be easily obtained by analyzing an asymptotic behaviour of the halforder impedance model (9) for low and high frequencies. Figure 7 shows the evolution of these parameters versus the polarization current $i$.

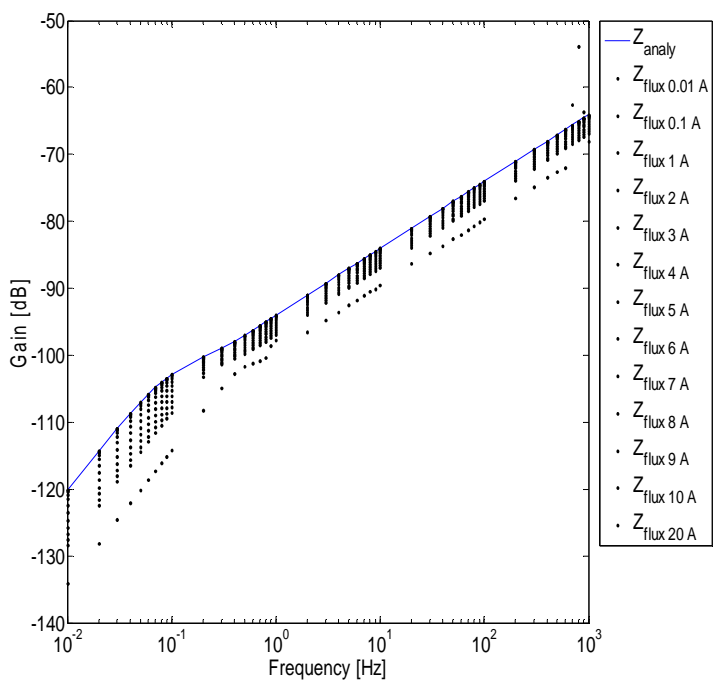

Fig.6. Big-signal model of the impedance
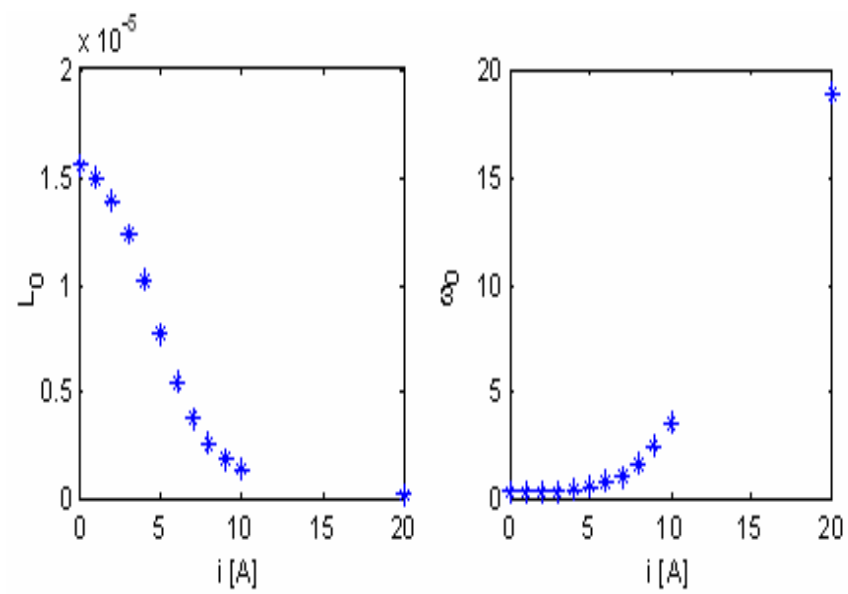

Fig.7. Parameters $L_{0}$ and $\omega_{0}$ identified from finite element simulations

It can be noticed, that the half-order impedance model is in very good agreement with simulations along a wide range of frequencies, as shown in figure 8 . This tends to prove that it could be used for modelling of synchronous generators operating in saturated conditions. 


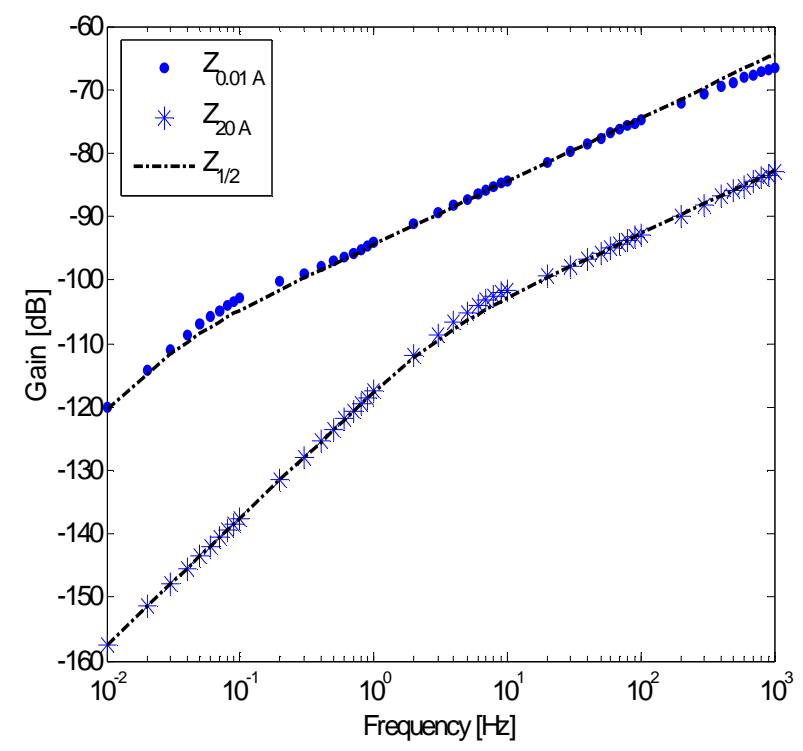

Fig. 8: Comparison of the half-order model with the finite element simulations

\section{SSFR TESTS OF SATURATED SYNCHRONOUS MACHINE}

The half-order impedance (9) is included in the equivalent circuits of a synchronous machine taking into account on the one hand eddy-currents in massive parts of the rotor or rotor bars and, on the other hand, saturation of the magnetic field. We consider then, that the parameters $L_{0}$ and $\omega_{0}$ are strongly influenced by saturation in low frequency range and the leakage inductance $l_{d}$ is influenced by saturation for high frequencies.

To identify these saturated equivalent circuits, the authors propose a method which consists in performing a regular SSFR with a short-circuited armature by a controlled DC voltage source (Fig. 9). This modified SSFR test in then easy to perform and allows precise control of the machine. This method is issued from works presented in [15].

However, this configuration allows obtaining only two operational transfer functions in $\mathrm{d}$ and $\mathrm{q}$ axes defined as the ratio between armature voltage and current in each axis.

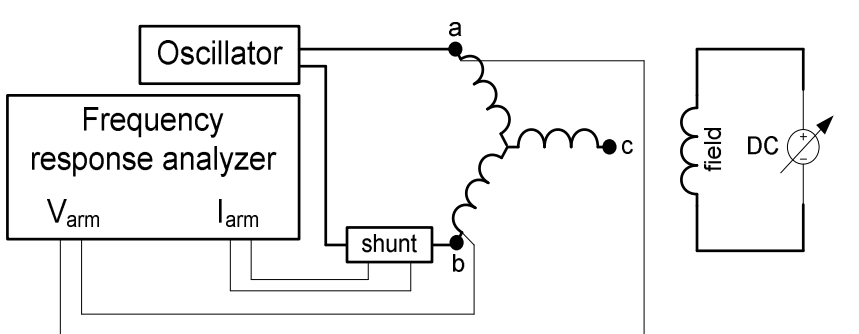

Fig. 9: Measurement setup for d-axis SSFR test of saturated synchronous machine
The test has been performed on a $3 \mathrm{kVA}$, 4-poles synchronous machine. Field current of the machine has been changed from 0 to 9 A. For each magnetization level, series of SSFR measurements have been done from $0.1 \mathrm{~Hz}$ to $1 \mathrm{kHz}$.

Identification of parameters has been done using Matlab ${ }^{\circledR}$ software using a Levenberg-Marquardt optimization algorithm (Isqnonlin function). A global identification is first obtained. Then, only parameter $L_{0}$ is identified another time from low frequency range for each magnetization level. The same approach is used to analyse the influence of saturation on $l_{d}$ and $\omega_{0}$.

As expected, the evolution of the parameters $L_{0}, l_{d}$, and $\omega_{0}$ (Fig. 10) is similar to the evolution of a saturated ferromagnetic sheet and is physically representative.
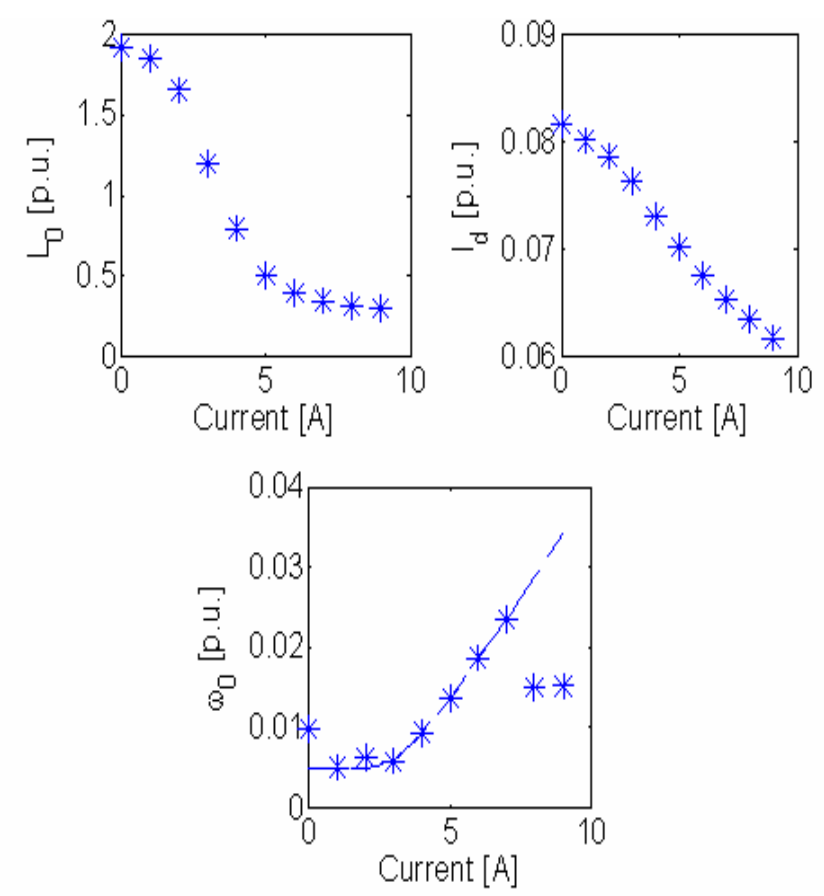

Fig. 10: Parameters $L_{1 d}, l_{d}$, and $\omega_{1 d}$ identified from the SSFR measurements of the saturated synchronous machine

Due to the numerical identification procedure and low measurement quality for high magnetization levels, a significant error in $\omega_{0}$ identification can be noticed. However, even after correction (represented with dashed line on figure 11 ), the model remains valid for all the magnetization levels and frequency ranges. 

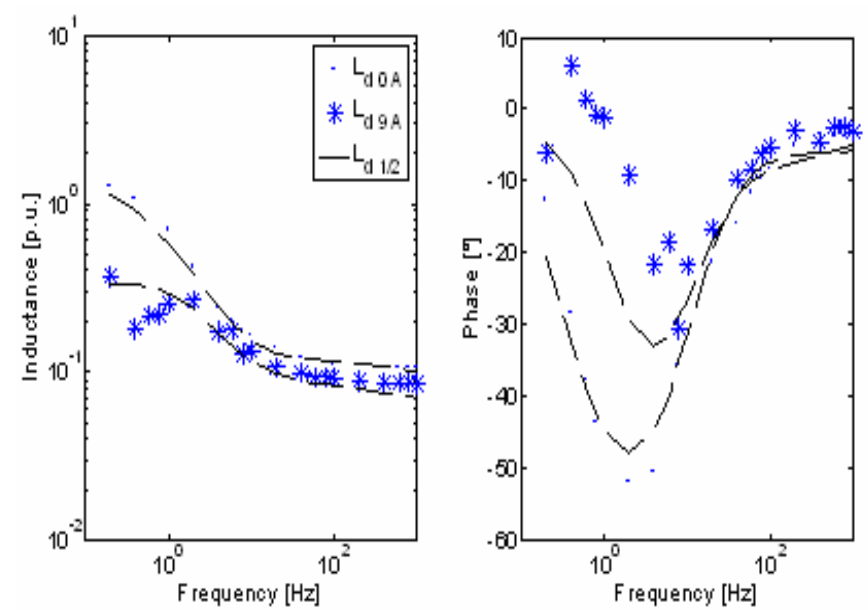

Fig. 11: Comparison of the half-order model with the SSFR measurements of the saturated synchronous machine

\section{CONCLUSION}

This paper presents an original knowledge model of a synchronous machine which takes into account of diffusion of the magnetic field in conductive parts of the machine and of magnetic saturation.

This half-order modelling has been validated with finite elements simulation in order to prove that it is well adapted to non-linear circuits. Then, the parameters of the non integer d-q generator equivalent circuits have been identified with data from the SSFR test performed on a $3 \mathrm{kVA}$ synchronous machine for different magnetization levels.

Future works will focus on robust control of this machine supplying an electrical system in saturated conditions. Then, the saturation would be considered as an uncertainty and control would have to respect performance templates whatever saturation level. For that purpose, the authors will also assess if non integer order controllers (i.e. controllers defined with non integer derivative or integrators) would also be an interesting way to improve the robustness of a system while keeping a few number of setting parameters [8].

\section{REFERENCES}

[1] S. Skogestad, I. Postlethwaite, Multivariable feedback control. Analysis and design. John Wiley \&sons. $2^{\text {nd }}$ edition. 2005

[2] I. Kamwa, P. Viarouge, H. Le-Hui, J. Dickinson, A frequency maximum likelihood of synchronous machine high order models using SSFR data, IEEE Trans. on Energy Conversion, Vol. 7, No.4, 1992, pp. 525-536.

[3] I.M. Canay, Causes of discrepancies on calculation of rotor quantities and exact equivalent diagrams of synchronous machine, IEEE Trans. on Power Apparatus, Vol. 88, 1969, pp. 1114-1120.

[4] D. Riu, N. Retière, M. Ivanès, Induced currents modelling by half-order systems. Application to hydro- and turbo-alternators, IEEE Trans. on Energy Conversion, Vol. 18, 2003, pp. 94-99.

[5] M.L. Awad, G.R.Slemon, M.R. Iravani, Distributed steady state nonlinear modelling of turboalternators, IEEE Trans. on Energy Conversion, Vol. 15, No. 3, 2000, pp. 233-239.

[6] A. M. El-Serafi, J. Wu, Determination of the parameters representing the cross-magnetizing effect in saturated synchronous machines, IEEE Trans. on Energy Conversion, Vol. 8, 1993, pp. 333-340.
[7] M.V.K Chari, P. Silvester, Analysis of turboalternator magnetic fields by finite elements, IEEE Trans., vol. PAS-90, No. 2, 1971, pp. 454-460

[8] A. Oustaloup \&al, A survey on the CRONE approach, Fractional differentiation and its applications, Part. 3, U Books, 2005

[9] S. Canat, J. Faucher, Modeling, identification and simulation of induction machine with fractional derivative, Fractional differentiation and its applications, Part. 2, U Books, 2005.

[10] E. Kuhn, C. Forgez, G. Friedrich, Fractional and diffusive representation of a $42 \mathrm{~V} \mathrm{Ni}-\mathrm{mH}$ battery, Fractional differentiation and its applications, Part. 2, U Books, 2005

[11] M. Usman Ifthikar, D. Riu, F. Druart, S. Rosini, Y. Bultel, N. Retière, "Dynamic modelling of proton exchange membrane fuel cell using noninteger derivatives", Journal of Power Sources, Vol. 160, Issue 2, October 2006, pp. 1170-1182

[12] P.L. Alger, Induction machines, Gordon and Breach, New-York, 1970.

[13] IEEE Std, Standard procedure for obtaining synchronous machine parameters by StandStill Frequency Response testing, IEEE Std 115A, 1995.

[14] I. Mayergoyz, Nonlinear diffusion of electromagnetic fields with applications to eddy-currents and superconductivity, Academic press, 1998

[15] J. Verbeeck, R.Pintelon, P. Lataire, Influence of saturation on estimated synchronous machine parameters in standstill frequency response tests, IEEE Trans. on Energy Conversion, Vol. 15, No. 3, 2000, pp. 277-283. 